\title{
PCCP
}

\section{A Model for the Effect of Bulk Liquid Viscosity on Acoustic Cavitation Bubble Dynamics}

\author{
Received, \\ Yang Shen ${ }^{\mathrm{a}, \mathrm{b}}$, Kyuichi Yasuic, Tong Zhü ${ }^{\mathrm{a} *}$, Muthupandian Ashokkumar ${ }^{\mathrm{b} *}$ \\ Accepted \\ DOI: \\ Abstract: A new model for single cavitation bubble dynamics has been developed to include the effect of bulk liquid viscosity \\ in addition to the effects of evaporation/condensation of water vapor, thermal conduction and the compressibility of liquid. \\ In this study, the liquid viscosity is divided into two parts: viscosity at the bubble interface ( $\left.\mu^{\prime}\right)$ and viscosity of the bulk \\ liquid $(\mu)$. A set of numerical calculations with and without $\mu$ have been performed at different viscosities $(0.001-0.014 \mathrm{~Pa}$ \\ s) to quantitatively analyze the effect of $\mu$ on single cavitation bubble dynamics. The results show that the effect can be \\ negligible for low viscosities, but it should be taken into account for relatively high viscosities.
}

\section{Introduction}

A gas bubble may undergo nonlinear oscillations in an acoustic field leading to acoustic cavitation under specific experimental conditions. ${ }^{1}$ High temperatures of thousands of degrees and high pressures of hundreds bars are generated inside the bubble as a result of strong quasi-adiabatic collapse. Under this extreme temperature condition, chemical reactions occur to generate highly reactive radicals within the collapsing bubble. ${ }^{2}$ Rayleigh $^{3}$ firstly solved the problem for the collapse of a spherically symmetric bubble in an incompressible non-viscous liquid. However, it appears that local velocities reach an appreciable fraction of the velocity of sound in the liquid, and the compressibility of the liquid cannot safely be neglected in many practical situations. Herring ${ }^{4}$ derived a better approximation, accurate to the first power of the ratio of liquid velocity to sonic velocity. His analysis, however, gives expressions for velocities and pressures only at the bubble wall and not throughout the liquid. Trilling ${ }^{5}$ obtained Herring's result using a simpler method, and also derived complicated integrals for obtaining the complete velocity and pressure field with firstorder accuracy. Plesset ${ }^{6}$ subsequently studied the dynamics of a vapor-filled bubble in a time-dependent pressure filed. Noltingk and Neppiras ${ }^{7}$ were the first to derive a differential equation (DE) for single cavitation bubble dynamics under the influence of a time-dependent pressure field. They took the liquid to be incompressible and the motions to be nondissipative, and solved their DE on a digital computer. Gilmore ${ }^{8}$ simplified the resulting expressions to forms which permit convenient numerical calculations including higher order

\footnotetext{
a. School of Mechanical Engineering and Automation, Northeastern University, 3-11 Wenhua Road, Heping District, Shenyang 110004, China.

b. School of Chemistry, University of Melbourne, VIC 3010, Australia.

c. National Institute of Advanced Industrial Science and Technology (AIST)

2266-98 Anagahora, Shimoshidami, Moriyama-ku, Nagoya 463-8560, Japan.

*Email: tongzhu@mail.neu.edu.cn

*Email:masho@unimelb.edu.au
}

compressibility terms and the effects of viscosity at the bubble interface and surface tension.

Several investigators have studied the effects of heat conduction, surface tension, liquid viscosity at the bubble interface $\left(\mu^{\prime}\right)$, compressibility of liquid and sound radiation on a single bubble under the influence of an acoustic field. ${ }^{6-10}$ However, a very few investigators have studied the effect of the bulk liquid viscosity $(\mu)$ on single bubble dynamics. It should be noted that the effect vanishes for low viscosities ${ }^{11}$, but there is no obvious evidence to proof this viewpoint and the effect of relatively large viscosities of bulk liquid $(\mu)$ has not been studied.

In this study, a new model for single cavitation bubble dynamics has been developed to understand the effect of viscosity. In addition to considering the effects of evaporation /condensation, and thermal conductivity of water and the compressibility of the liquid, liquid viscosity at the bubble interface and bulk liquid are taken into account. Numerical calculations have been carried out to quantitatively analyse the effect of bulk liquid viscosity on single cavitation bubble dynamics.

\section{Model}

A schematic diagram depicting the temperature profile both inside and outside a single bubble is illustrated in Fig. 1 . In this model, the following assumptions have been made: (1) The bubble collapse is spherically symmetric, (2) The effects of gravity and gas diffusion are negligible, (3) The pressure inside the bubble is spatially uniform, (4) The temperature inside the bubble $(T)$ is spatially uniform except for the thin boundary layer $(n \lambda)$, as shown in Figure 1, (5) Some physical properties of liquid are constant, such as, the density $\rho$, the ambient pressure $p_{0}$, the sound speed in the liquid $c_{0}$ and (6) The amount of gas (air) inside a bubble is constant. ${ }^{1}$ 
According to the assumption (4), the thickness of the thin boundary layer is $n \lambda$, where $n$ is a constant and $\lambda$ is the mean free path of a gas molecule. Schematic diagram depicting the

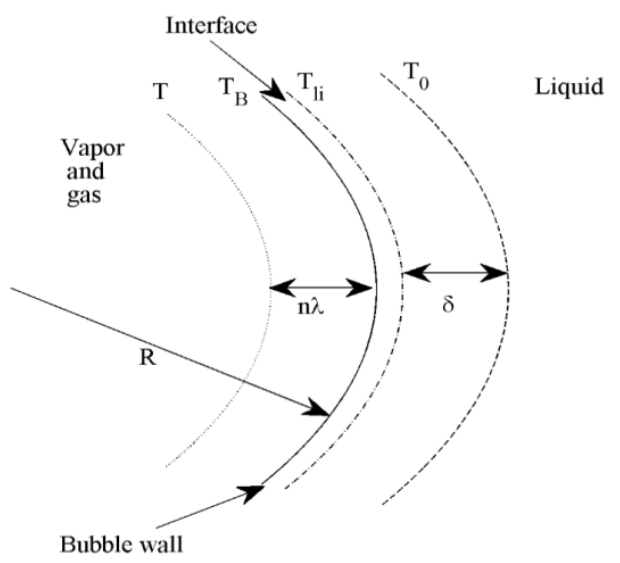

Fig. 1 Schematic diagram depicting the temperature profile. Reused from Shen et al., Copyright (2015), with permission from Elsevier. [Ref. 1]

temperature profile is shown in Fig. 1.

The temperature distribution ( $T$ ) inside the bubble is assumed to be

$$
\begin{aligned}
& T=T, \quad 0 \leq r \leq R-n \lambda \\
& T=T_{B}-\left.\frac{\partial T}{\partial r}\right|_{r=R}(r-R), \quad R-n \lambda \leq r \leq R,
\end{aligned}
$$

where $T_{B}$ is the gas temperature at bubble wall.

Temperature jump $(\Delta T)$ exists at the bubble wall as predicted by the kinetic theory of gases ${ }^{12-14}$ :

$$
T_{B}=T_{l i}+\Delta T
$$

where $T_{l i}$ is the liquid temperature at the bubble wall, the temperature jump ( $\Delta T)$ :

$\Delta T=-\frac{s}{2 k} \sqrt{\frac{\pi m^{\prime}}{k T_{B}}} \frac{2-a^{\prime} \alpha_{e}}{\alpha_{e}} \kappa \frac{T_{B}-T}{n}$

where $k$ is the Boltzmann constant, $m^{\prime}$ is the mean mass of a molecule, $a^{\prime}$ is a constant, $\kappa$ is the thermal conductivity of the gas, $s$ is the cross section of a molecule in the bubble.

As an equation of bubble radius ( $R$ ), Eq.(4), is employed, in which compressibility of liquid, effect of evaporation and condensation of water vapor at the bubble wall and the effect of the liquid viscosity on the momentum equation are taken into account.

$$
\begin{aligned}
& \left(1-\frac{\dot{R}}{c_{\infty}}+\frac{\dot{m}}{c_{\infty} \rho_{l}}\right) R\left(\ddot{R}-\frac{\ddot{m}}{\rho_{l}}\right)+\frac{3}{2} \dot{R}^{2}\left(1-\frac{\dot{R}}{3 c_{\infty}}+\frac{\dot{m}}{3 c_{\infty} \rho_{l}}\right) \\
& =\frac{1}{\rho_{l}}\left(1+\frac{\dot{R}}{c_{\infty}}\right)\left[p_{B}-p_{s}\left(t+\frac{R}{c_{\infty}}\right)-p_{0}\right]+\frac{\dot{m}}{\rho_{l}}\left(\dot{R}+\frac{\dot{m}}{2 \rho_{l}}+\frac{\dot{m} \dot{R}}{2 c_{\infty} \rho_{l}}\right) \\
& +\left[\frac{R}{c_{\infty} \rho_{l}}+\frac{4 \mu}{3 c_{\infty}^{2} \rho_{l}^{2}}\left(1+\frac{\dot{R}}{c_{\infty}}\right)\right] \frac{d p_{B}}{d t}+\frac{4 \mu R}{3 c_{\infty}^{3} \rho_{l}^{2}} \frac{d^{2} p_{B}}{d t^{2}}
\end{aligned}
$$

where $\cdot$ denotes the time derivate, $t$ is the time, $\rho_{l}$ is the liquid density, $p_{l}$ is the pressure in the liquid. $\mu$ is the viscosity of the liquid from the bulk of liquid, $\dot{m}$ is the net rate of evaporation (condensation) per unit and unit time.
The liquid pressure on the external side of the bubble wall $p_{B}$ is related to the pressure inside the bubble $\left(p_{g}(t)\right)$ :

$P_{B}=P_{g}(t)-\frac{2 \sigma}{R}-\frac{4 \mu^{\prime}}{R}\left(\dot{R}-\frac{\dot{m}}{\rho}\right)-\dot{m}^{2}\left(\frac{1}{\rho}-\frac{1}{\rho_{g}}\right)-\frac{4 \mu}{3 c^{2} \rho_{l}} \frac{d P_{B}}{d t}$

where $\sigma$ is the surface tension, $\mu^{\prime}$ is the liquid viscosity at the bubble interface, and $\rho_{g}$ is the density inside the bubble. Note that the third term of eq.(5) includes the liquid viscosity at the bubble interface, the fifth term of eq.(5) includes bulk liquid viscosity.

The rate of evaporation and condensation $\dot{m}$ is expressed as followed ${ }^{15}$ :

$\dot{m}=\frac{\alpha_{M}}{\sqrt{2 \pi R_{v}}}\left(\frac{p_{v}^{*}}{\sqrt{T_{l i}}}-\frac{\Gamma p_{v}}{\sqrt{T_{B}}}\right)$

where $\alpha_{M}$ is the accommodation coefficient for evaporation or condensation, $p_{v}$ is the actual vapor pressure, $p_{v}^{*}$ is the saturated vapor pressure. $R_{v}$ is the gas constant of the vapor. The correction factor $\Gamma$ is expressed as follows:

$\Gamma=\exp \left(-\Omega^{2}\right)-\Omega \sqrt{\pi}\left(1-\frac{2}{\sqrt{\pi}} \int_{0}^{\Omega} \exp \left(-x^{2}\right) d x\right)$

$$
\text { where } \quad \Omega=\frac{\dot{m}}{p_{v}}\left(\frac{R_{v} T}{2}\right)^{1 / 2} \text {. }
$$

The pressure inside the bubble $\left(p_{g}(t)\right)$ can be obtained from the van der Waals equation:

$$
p_{g}(t)=\frac{R_{g} T}{v-b}-\frac{a}{v(v+b)}
$$

where $R_{g}$ is the gas constant, $v$ is the molar volume, $a$ and $b$ are the van der Waals constants.

The gas temperature inside the bubble $(T)$ can be expressed as followed:

$$
T=\frac{N_{A}^{2} E V+\left(n_{\mathrm{H}_{2} \mathrm{O}}+n_{\text {air }}\right)^{2} a}{\left(n_{\text {air }} C_{v, \text { air }}+n_{\mathrm{H}_{2} \mathrm{O}} C_{v, \mathrm{H}_{2} \mathrm{O}}\right) N_{A} V}
$$

where $N_{A}$ is the Avogadro number, $V$ is the bubble volume, $E$ is the internal energy of the bubble, $n$ is the amount of molecule, $C_{v}$ is the heat capacity at constant volume, the suffix air and $\mathrm{H}_{2} \mathrm{O}$ represent the air and water vapor, respectively.

The change of the thermal energy of a bubble $(\Delta E)$ in time $(\Delta t)$ is expressed by ${ }^{11}$

$$
\Delta E(t)=-p_{g}(t) \Delta V(t)+\frac{N_{A}}{M_{\mathrm{H}_{2} \mathrm{O}}} 4 \pi R^{2} \dot{m} e_{\mathrm{H}_{2} \mathrm{O}} \Delta t+\left.4 \pi R^{2} \kappa \frac{\partial T}{\partial r}\right|_{r=R} \Delta t
$$

The amount of the water vapor molecule $n_{H_{2} \mathrm{O}}$ can be expressed:

$$
n_{\mathrm{H}_{2} \mathrm{O}}(t+\Delta t)=n_{\mathrm{H}_{2} \mathrm{O}}(t)+\frac{N_{\mathrm{A}}}{M_{\mathrm{H}_{2} \mathrm{O}}} 4 \pi R^{2} \dot{m} \Delta t
$$

where $M_{\mathrm{H}_{2} \mathrm{O}}$ is the molar mass of the water vapor, $e_{\mathrm{H}_{2} \mathrm{O}}$ is the energy carried by an evaporating or condensing vapor molecule, which is expressed by

$$
e_{\mathrm{H}_{2} \mathrm{O}}=\frac{C_{v, \mathrm{H}_{2} \mathrm{O}}}{N_{\mathrm{A}}} T_{B} \text {. }
$$

The description of the variation of liquid temperature at the bubble wall is as follows. Eq.(12) can be obtained from Continuity of energy flux at the bubble wall ${ }^{12}$ : 


$$
\left.\kappa_{l} \frac{\partial T_{1}}{\partial r}\right|_{r=R}=\left.\kappa \frac{\partial T}{\partial r}\right|_{r=R}+\dot{m} L+\frac{\dot{m}}{M_{H_{2} \mathrm{O}}} C_{V, H_{2} \mathrm{O}} \Delta T
$$

where $\kappa_{l}$ is the thermal conductivity of the liquid, $T_{l}(r)$ is the liquid temperature at radius $r$ from the center of the bubble outside the bubble.

The spatial distribution of the liquid temperature should satisfy the boundary conditions as follows:

$$
\begin{aligned}
& T_{l}(r=R)=T_{l i} ; \\
& \left.\frac{\partial T_{l}(r)}{\partial r}\right|_{r=R}=\left.\frac{\partial T_{l}}{\partial r}\right|_{r=R} ; \\
& T_{l}(r=R+\delta)=T_{0} .
\end{aligned}
$$

From the boundary conditions, the temperature profile of the thin liquid layer outside of the bubble can be obtained. The details can be obtained in reference ${ }^{1}$.

The liquid temperature at the bubble wall $\left(T_{l i}\right)$ can be expressed by Eq. $(14)^{12}$ :

$$
T_{l i}(t+\Delta t)=T_{l i}(t)+\frac{4 \pi R^{2} j_{1} \Delta t-4 \pi(R+\delta)^{2} j_{2} \Delta t}{\frac{4}{3} \pi\left[(R+\delta)^{3}-R^{3}\right] \rho c_{p}}
$$

where $j_{1}\left(j_{2}\right)$ is the energy flux at $r=R(r=R+\delta)$ per unit area and unit time, $c_{p}$ is the specific heat of liquid water at constant pressure. $j_{1}$ and $j_{2}$ are expressed by ${ }^{12}$

$$
\begin{aligned}
& j_{1}=-\left.\left.\kappa_{l}\right|_{r=R} \frac{\partial T_{l}}{\partial r}\right|_{r=R} \\
& j_{2}=-\left.\left.\kappa_{l}\right|_{r=R+\delta} \frac{\partial T_{l}}{\partial r}\right|_{r=R+\delta}
\end{aligned}
$$

\section{Numerical Simulation and Results}

Calculations were carried out at time $t=0$ under the same parameters as in reference 16: the initial bubble radius $R_{0}=4.5 \mu \mathrm{m}$; the ultrasonic frequency $f=26.5 \mathrm{kHz}$; the ultrasonic amplitude are $P_{a}=1.325 \mathrm{~atm}$; the sound speed in liquid $c=1481 \mathrm{~m} / \mathrm{s}$; the viscosity of liquid $\mu^{\prime}=\mu=0.007 \mathrm{~Pa} \square \mathrm{s}$.

The numerical simulations and results for one acoustic cycle are shown in Fig.2. Every horizontal axis in Fig. 2 is the time axis corresponding to about $38 \mu \mathrm{s}$. The numerically calculated result of the bubble radius (line) and the experimental data 16 (circles) are shown in Fig. 2(a). It is clearly seen that the numerically calculated result fits well with the experimental data. The velocity of the bubble wall is shown in Fig. 2(b). It can be seen that the velocity is less than the sound speed in the gas inside the bubble. On bubble collapse, the maximum of the velocity $(|\dot{R}|)$ can be up to $1060.6 \mathrm{~m} / \mathrm{s}$ which is less than the sound speed inside the bubble calculated by $\sqrt{d p / d \rho_{g}}(1711.5$ $\mathrm{m} / \mathrm{s})$ at the same time, so the Mach number $M=|\dot{R}| / c$ is less than one in the whole period of the cavitation bubble collapse. Thus, the assumption (3) "The pressure inside the bubble is spatially uniform" is valid even when the bubble collapse occurs strongly. Hence, the numerical simulations and results obtained for strong bubble collapse conditions are accurate from quantitative and qualitative view points. The temperature inside the bubble $(T)$ is shown in Fig. 2(c). It can be seen that the temperature $(T)$ inside the bubble slightly decreases in the growth phase (almost equal to the liquid temperature $\left(T_{0}\right)$ ). At the collapse phase of the bubble, the temperature $(T)$ increases suddenly to about $7700 \mathrm{~K}$. The liquid temperature at the external of the bubble wall $\left(T_{l i}\right)$ is in Fig. $2(\mathrm{~d})$. It can be seen that the tendency of $T_{l i}$ is consistent with that of the temperature inside the bubble and the variation of $T_{l i}$ is not obvious except at the collapse and at the first two rebound phases. At the strong collapse, $T_{l i}$ increases dramatically up to about $1760 \mathrm{~K}$. To some extent, the maximum of $T$ and $T_{l i}$ match with that reported by Suslick ${ }^{17}$. The pressure inside the bubble $\left(p_{g}\right)$ is shown in Fig. 2(e). It can be seen that the pressure $p_{g}$ decreases at the expansion phase and increases suddenly at the collapse phase, followed by some small oscillations because of the rebounds of the bubble. The maximum and minimum pressures are $3 \mathrm{GPa}$ and $2436 \mathrm{~Pa}$, respectively. The number of molecules of water vapor, air and the total molecules inside the bubble are shown in Fig. 2(f). The solid, the dashed and the dashed-dot lines are the number of water vapor, air molecules and total molecules, respectively. It can be seen that a large number of water vapor molecules enter into the bubble at the expansion phase. About $92 \%$ of the total contents is water vapor at the maximum bubble radius. The number of water vapor molecules decreases significantly at the collapse phase of the bubble.

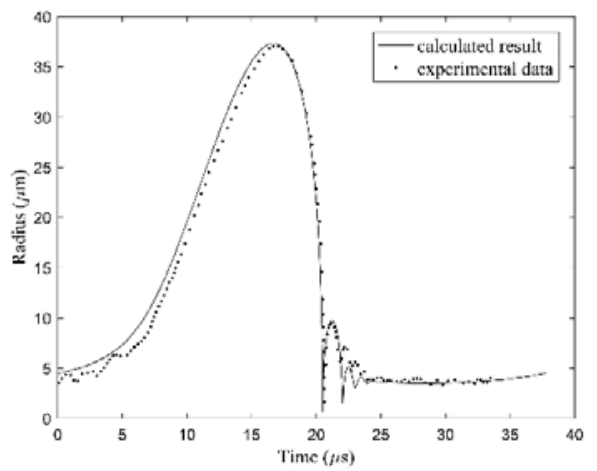

(a)

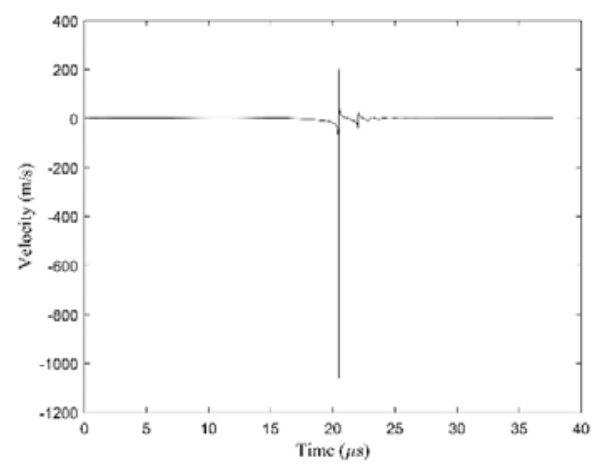

(b) 


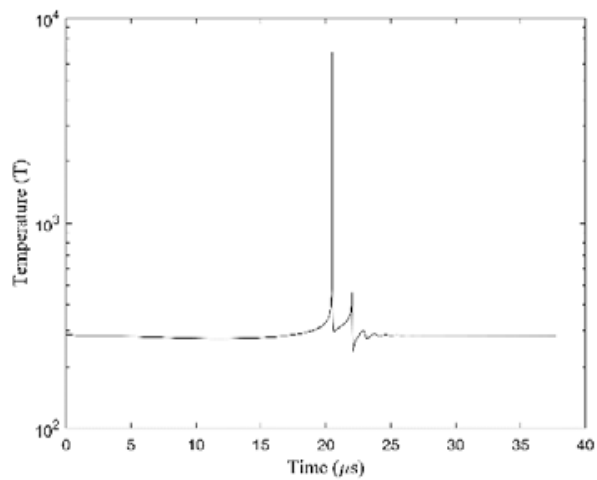

(c)

Fig. 2 Calculated results of a single bubble for one acoustic cycle

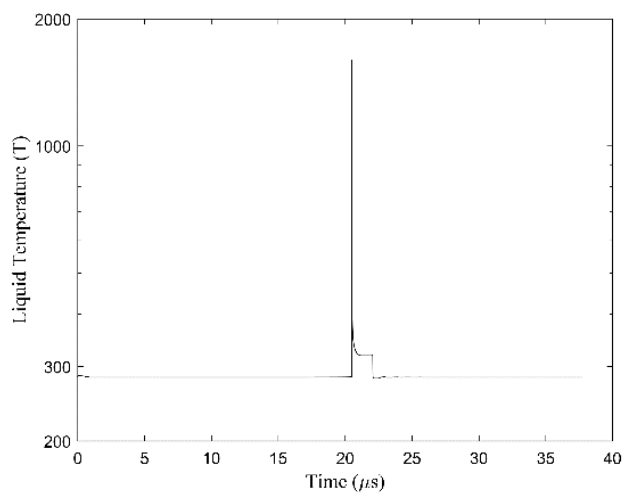

(d)

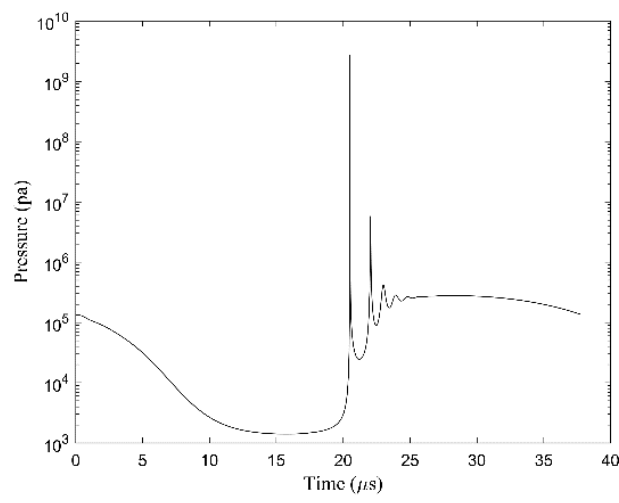

(e)

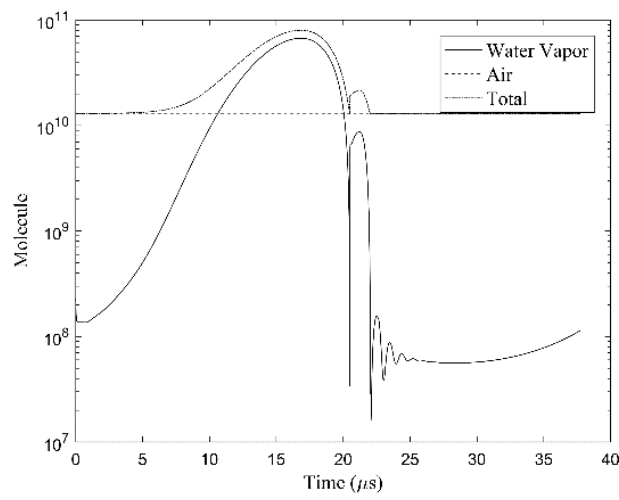

(f)
Fig. 2 (continued) Calculated results of a single bubble for one acoustic cycle

As mentioned in Section I, only a few studies have focused on the effect of bulk liquid viscosity $(\mu)$ on single bubble dynamics. In order to study the effect of bulk liquid viscosity $(\mu$ ) on single bubble dynamics (the term including $\mu$ in equations (4) and (5)) , a set of numerical calculations with or without the effect of $\mu$ were carried out (the ultrasonic frequency and the ultrasonic amplitude are $26.5 \mathrm{kHz}$ and $1.325 p_{0}$, respectively).

The maximum temperature and pressure inside the bubble under different viscosities (0.001-0.014 $\mathrm{Pa}$ s) are shown in Fig.3(a) and Fig.3(b), respectively. It can be seen that the maximum temperature and pressure inside the bubble with the effect of $\mu$ are larger than those without including the viscosity effect. The difference increases with an increase in viscosity. The effects for the maximum temperature and pressure are

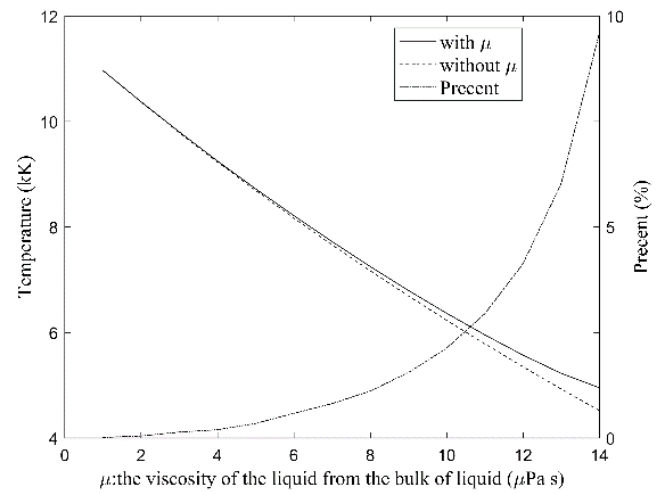

(a)

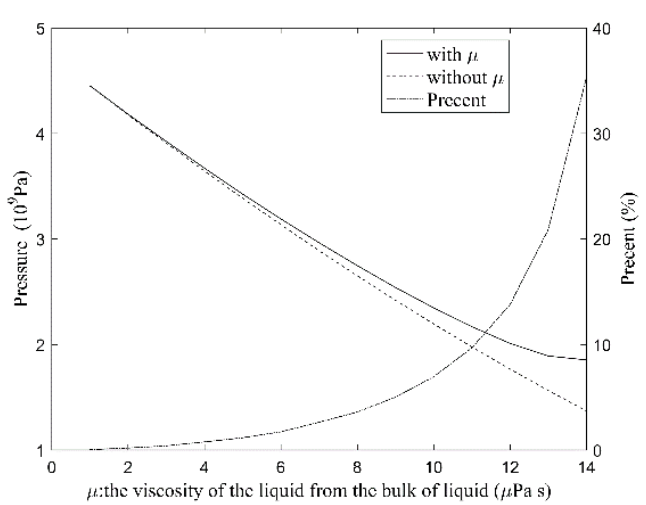

(b)

Fig. 3 The maximum temperature and pressure inside the bubble under different viscosities of the liquid from the bulk of liquid $\mu$

insignificant when the viscosity is $0.001 \mathrm{~Pa}$ s. The effects on maximum temperature and pressure are about $10 \%$ and $35 \%$, respectively when the viscosity is $0.014 \mathrm{~Pa}$ s. These results show that the effect can be neglected for low viscosities which is consistent with that reported earlier ${ }^{11}$. However, the current study clearly shows that viscosity effect cannot be ignored at high viscosities.

\section{Conclusion}


A set of equations for single cavitation bubble dynamics are newly derived from the Navier-Stokes equations, which are different from those reported in previous studies to include the effect of bulk liquid viscosity $\mu$. A set of numerical calculations with or without the bulk liquid viscosity term have been completed to quantitatively analyse the effect of bulk liquid viscosity on single cavitation bubble dynamics. The results show that the effect on the maximum temperature and pressure inside the bubble on bubble collapse can be negligible for low viscosities, but significant for relative high viscosities.

\section{Acknowledgments}

This paper is supported by National Natural Science Foundation of China (51178089), Zhu Tong doctoral fund (20130042110009) and the Fundamental Research Funds for the Central Universities (N150304002, N1503040016) and is funded by CSC.

\section{Appendix A: Derivation of Eq.(4)}

Under the assumptions in section II, the governing balance equations of the liquid and the boundary conditions at the interface may be expressed as follows.

Total mass balance:

$$
\frac{\partial \rho_{l}}{\partial t}+\frac{\partial}{\partial r}\left(\rho_{l} u_{l}\right)+\frac{2 \rho_{l} u_{l}}{r}=0
$$

Momentum balance equation:

$$
\frac{\partial u_{l}}{\partial t}+u_{l} \frac{\partial u_{l}}{\partial r}+\frac{1}{\rho_{l}} \frac{\partial p_{l}}{\partial r}=\frac{4 \mu}{3 \rho_{l}}\left(\frac{\partial u_{l}}{\partial r}+\frac{2}{r} u_{l}\right)_{r}
$$

The boundary condition:

$$
\rho_{l}\left(\left(\frac{\partial \varphi}{\partial r}\right)_{R}-\dot{R}\right)=-\dot{m}
$$

where $u_{l}$ is the local radial velocity in the liquid, $r$ is the radial coordinate, $\varphi$ is the velocity potential of the liquid,

Using $\varphi$ instead of $u_{1}$, eqs.(A.1) and (A.2) are rewritten:

$$
\begin{aligned}
& \frac{\partial \rho_{l}}{\partial t}+\rho_{l}\left(\frac{\partial^{2} \phi}{\partial r^{2}}+\frac{2}{r} \frac{\partial \phi}{\partial r}\right)+\frac{\partial \phi}{\partial r} \frac{\partial \rho_{l}}{\partial r}=0 \\
& \frac{\partial^{2} \phi}{\partial r \partial t}+\frac{\partial \phi}{\partial r} \frac{\partial^{2} \phi}{\partial r^{2}}+\frac{1}{\rho_{l}} \frac{\partial p_{l}}{\partial r}=\frac{4 \mu}{3 \rho_{l}}\left(\frac{\partial^{2} \phi}{\partial r^{2}}+\frac{2}{r} \frac{\partial \phi}{\partial r}\right)_{r}
\end{aligned}
$$

Integrating eq.(A.5) by $r$ yields

$$
\frac{\partial \varphi}{\partial t}+\frac{1}{2}\left(\frac{\partial \varphi}{\partial r}\right)^{2}+\frac{p_{l}-p_{\infty}}{\rho_{l}}=\frac{4 \mu}{3 \rho_{l}}\left(\frac{\partial^{2} \varphi}{\partial r^{2}}+\frac{2}{r} \frac{\partial \varphi}{\partial r}\right)
$$

Form the eq.(A.4), the term in brackets of the right hand of eq.(A.6):

$$
\begin{aligned}
\frac{\partial^{2} \varphi}{\partial r^{2}}+\frac{2}{r} \frac{\partial \varphi}{\partial r} & =\frac{\partial u_{l}}{\partial r}+\frac{2}{r} u_{l}=-\frac{1}{\rho_{l}}\left(\frac{\partial \rho_{l}}{\partial t}+u_{l} \frac{\partial \rho_{l}}{\partial r}\right) \\
& =-\frac{1}{\rho_{l}} \frac{d \rho_{l}}{d t}=-\frac{1}{\rho_{l}} \frac{d \rho_{l}}{d p_{l}} \frac{d p_{l}}{d t}=-\frac{1}{c^{2} \rho_{l}} \frac{d p_{l}}{d t}
\end{aligned}
$$

where $\mathrm{c}$ is the speed of sound in liquid, $p_{\infty}$ is the undisturbed static pressure in the liquid.

According to the reference ${ }^{12}$, with $f$ and $g$ arbitrary functions: $\varphi=-\frac{f\left(t-r / c_{\infty}\right)}{r}-\frac{g\left(t+r / c_{\infty}\right)}{r}$

From eqs.(A.3) and (A.8):

$\frac{f^{\prime}}{R}=c_{\infty}\left(\dot{R}-\frac{\dot{m}}{\rho_{l}}+\frac{\varphi(R)}{R}\right)+\frac{g^{\prime}}{R}$

From eqs. (A.8) and (A.9):

$\left(\frac{\partial \varphi}{\partial t}\right)_{R}=-C_{\infty}\left(\dot{R}-\frac{\dot{m}}{\rho_{l}}+\frac{\varphi(R)}{R}\right)-\frac{2 g^{\prime}}{R}$

Inserting eqs.(A.3), (A.7) and (A.10) into eq. (A.6) yields

$-c_{\infty}\left(\dot{R}-\frac{\dot{m}}{\rho_{l}}+\frac{\varphi(R)}{R}\right)-\frac{2 g^{\prime}}{R}+\frac{1}{2}\left(\dot{R}-\frac{\dot{m}}{\rho_{l}}\right)^{2}+\frac{p_{B}-p_{\infty}}{\rho_{l}}=-\frac{4 \mu}{3 \rho_{l}} \frac{1}{c_{\infty}^{2} \rho_{l}} \frac{d p_{B}}{d t}$

Multiplying eq. (A.11) by $R$ and differentiating by $t$ yields

$$
\begin{aligned}
& -c_{\infty} \dot{R}\left(\dot{R}-\frac{\dot{m}}{\rho_{l}}\right)-c_{\infty} R\left(\ddot{R}-\frac{\ddot{m}}{\rho_{l}}\right)-c_{\infty}\left(\frac{d \phi}{d t}\right)_{R}-2 \frac{d g^{\prime}}{d t} \\
& +\frac{1}{2} \dot{R}\left(\dot{R}-\frac{\dot{m}}{\rho_{l}}\right)^{2}+R\left(\dot{R}-\frac{\dot{m}}{\rho_{l}}\right)\left(\ddot{R}-\frac{\ddot{m}}{\rho_{l}}\right)+ \\
& \frac{\dot{R}\left(p_{B}-p_{0}\right)}{\rho_{l}}+\frac{R}{\rho_{l}} \frac{d p_{B}}{d t}+\frac{4 \mu}{3 c_{\infty}{ }^{2} \rho_{l}^{2}}\left(\dot{R} \frac{d p_{B}}{d t}+R \frac{d^{2} p_{B}}{d t^{2}}\right)=0
\end{aligned}
$$

From eqs.(A.3), (A.6) and (A.7),

$$
\begin{aligned}
& \left(\frac{d \varphi}{d t}\right)_{R}=\left(\frac{\partial \varphi}{\partial t}+\frac{\partial \varphi}{\partial r} \frac{d r}{d t}\right)_{R}= \\
& -\frac{1}{2}\left(\dot{R}-\frac{\dot{m}}{\rho_{l}}\right)^{2}-\frac{p_{B}-p_{\infty}}{\rho_{l}}+\left(\dot{R}-\frac{\dot{m}}{\rho_{l}}\right) \dot{R}-\frac{4 \mu}{3 \rho_{l}} \frac{1}{c_{\infty}{ }^{2} \rho_{l}} \frac{d p_{B}}{d t}
\end{aligned}
$$

When the incident field is a plane wave with angular frequency $\omega$ and pressure amplitude $p_{A}{ }^{18}$,

$$
2 g^{\prime \prime}(t)=\frac{p_{A} c_{\infty}}{\rho_{l}} \sin \omega t=\frac{c_{\infty}}{\rho_{l}} p_{s}\left(t+\frac{R}{C_{\infty}}\right)
$$

From eqs.(A.12), (A.13) and (A.14),

$$
\begin{aligned}
& \left(1-\frac{\dot{R}}{c_{\infty}}+\frac{\dot{m}}{c_{\infty} \rho_{l}}\right) R\left(\ddot{R}-\frac{\ddot{m}}{\rho_{l}}\right)+\frac{3}{2} \dot{R}^{2}\left(1-\frac{\dot{R}}{3 c_{\infty}}+\frac{\dot{m}}{3 c_{\infty} \rho_{l}}\right) \\
& =\frac{1}{\rho_{l}}\left(1+\frac{\dot{R}}{c_{\infty}}\right)\left[p_{B}-p_{s}\left(t+\frac{R}{c_{\infty}}\right)-p_{0}\right]+\frac{\dot{m}}{\rho_{l}}\left(\dot{R}+\frac{\dot{m}}{2 \rho_{l}}+\frac{\dot{m} \dot{R}}{2 c_{\infty} \rho_{l}}\right) \\
& +\left[\frac{R}{c_{\infty} \rho_{l}}+\frac{4 \mu}{3 c_{\infty}{ }^{2} \rho_{l}^{2}}\left(1+\frac{\dot{R}}{c_{\infty}}\right)\right] \frac{d p_{B}}{d t}+\frac{4 \mu R}{3 c_{\infty}{ }^{3} \rho_{l}^{2}} \frac{d^{2} p_{B}}{d t^{2}}
\end{aligned}
$$

where $P_{B}$ is the liquid pressure on the external side of the bubble wall ${ }^{15}$ :

$$
p_{B}=p_{g}-\frac{2 \sigma}{R}+\left[\rho_{g}\left(u_{m}-\dot{R}\right)\left(u_{m}-u_{l}\right)\right]_{R}+\frac{4 u_{l}}{3}\left(\frac{\partial u_{l}}{\partial r}-\frac{u_{l}}{r}\right)_{R}
$$

According to the Continuity equations ${ }^{15}$ :

$$
\begin{aligned}
& {\left[\rho_{l}\left(u_{l}-\dot{R}\right)\right]=-\dot{m}} \\
& {\left[\rho_{m}\left(u_{m}-\dot{R}\right)\right]_{R}=-\dot{m}}
\end{aligned}
$$

Inserting (A.7) and (A.17) into the last term of (A.16) yields $\left(\frac{\partial u_{l}}{\partial r}-\frac{u_{l}}{r}\right)_{R}=\left(\frac{\partial u_{l}}{\partial r}+\frac{2 u_{l}}{r}-\frac{3 u_{l}}{r}\right)_{R}=-\frac{1}{c^{2} \rho_{l}} \frac{d p_{B}}{d t}-\frac{3}{R}\left(\dot{R}-\frac{\dot{m}}{\rho_{l}}\right)$

Inserting (A.17), (A.18) and (A.19) into (A.16) yields

$$
P_{B}=P_{g}(t)-\frac{2 \sigma}{R}-\frac{4 \mu}{R}\left(\dot{R}-\frac{\dot{m}}{\rho}\right)-\dot{m}^{2}\left(\frac{1}{\rho}-\frac{1}{\rho_{g}}\right)-\frac{4 \mu}{3 c^{2} \rho_{l}} \frac{d P_{B}}{d t}
$$




\section{Reference}

1 Y. Shen, K. Yasui, Z. Sun, B. Mei, M. You, T. Zhu, Study on the spatial distribution of the liquid temperature near a cavitation bubble wall, Ultrason. Sonochem. 29 (2016) 394-400.

2 M. Ashokkumar, The characterization of acoustic cavitation bubbles - An overview, Ultrason. Sonochem. 18 (2011) 864872.

3 Lord Rayleigh, On the pressure developed in a liquid during the collapse of a spherical cavity, Philos. Mag. Ser. 6.34 (1917) 94-98.

4 C. Herring, Theory of the pulsations of the gas bubble produced by an underwater explosion, OSRD Report. 236 (1941).

5 L. Trilling, The collapse and rebound of a gas bubble, J. Appl. Phys. 23 (1952) 14-17.

6 M. S. Plesset, The dynamics of cavitaion bubbles, J. Appl. Mech. 16 (1949) , 277-282.

7 B. E. Noltingk, E. A. Neppiras, Cavitation produced by Ultrasonics, Proc. Phys. Soc. Sect. B. 63 (1950) 674-685.

8 F. R. Gilmore, The growth or collapse of a spherical bubble in a viscous compressible liquid, Calif. Inst. Tech Eng. Rep. 26 (1952) 1-40.

9 H. G. Flynn, Cavitation dynamics. I. A mathematical formulation, J. Acoust. Soc. Am. 57(1975), 1379-1396.

10 C. Cogné, S. Labouret, R. Peczalski, O. Louisnard, F. Baillon, F. Espitalier, Theoretical model of ice nucleation induced by acoustic cavitation. Part 1: Pressure and temperature profiles around a single bubble, Ultrason. Sonochem. 29 (2016) 447454.

$11 \mathrm{~J}$. Holzfuss, Acoustic energy radiated by nonlinear spherical oscillations of strongly driven bubbles, Proc. R. Soc. A Math. Phys. Eng. Sci. 466 (2010) 1829-1847.

$12 \mathrm{~K}$. Yasui, Variation of Liquid temperature at bubble wall near the Sonoluminescence threshold, J.Phys.Soc.Jpn. 65(9) (1996) 2830-2840.

$13 \mathrm{~K}$. Yasui, Effects of thermal conduction on bubble dynamics near the sonolumiescence threshold, J.Acoust.Soc.Am. 98(5) (1995) 2772-2782.

$14 \mathrm{~K}$. Yasui, Alternative model of single-bubble sonoluminecence, Phys Rev E. 56(6) (1997) 6750-6760.

$15 \mathrm{~S}$. Fujikawa, T. Akamatsu, Effect of the non-equilibrium condensation of vapor on the pressure wave produced by the collapse of the bubble in a liquid, J.Fluid Mech. 97(3) (1980) 481-512.

16 B. P. Barber, S. J. Putterman. Light scattering measurements of the repetitive supersonic imiplosion of a sonoluminescing bubble. Physical Review Letters, APS, 69(26) (1992) 38393842.

17 K. S. Suslick, D. A. Hammerton, R. E. Cline. The Sonochemical Hot. J. Am. Chem. Soc, 108(7) (1986): 5641-5642.

18 J. B. Keller, Bubble oscillations of large amplitude, J Acoust Soc Am. 68(2) (1980) 628-633. 


\section{University Library}

\section{- M M N E R VA A gateway to Melbourne's research publications}

Minerva Access is the Institutional Repository of The University of Melbourne

Author/s:

Shen, Y;Yasui, K;Zhu, T;Ashokkumar, M

Title:

A model for the effect of bulk liquid viscosity on cavitation bubble dynamics

Date:

2017-08-21

Citation:

Shen, Y., Yasui, K., Zhu, T. \& Ashokkumar, M. (2017). A model for the effect of bulk liquid viscosity on cavitation bubble dynamics. PHYSICAL CHEMISTRY CHEMICAL PHYSICS, 19 (31), pp.20635-20640. https://doi.org/10.1039/c7cp03194g.

Persistent Link:

http://hdl.handle.net/11343/241896 